Araştırma Makalesi - Research Article

\title{
Modifiye Edilmiş Coulomb Potansiyelli Conformable Sturm-Liouville Problemi
}

\author{
Erdal Baş ${ }^{1 *}$, Isam Najemadeen ${ }^{2}$ \\ Geliş / Received: 31/12/2019 \\ Revize / Revised: 13/06/2020 \\ Kabul / Accepted: 25/06/2020 \\ ÖZ
}

Bu makalede, modifiye edilmiş Coloumb Potansiyele sahip Sturm Liouville probleminin uyumlu mertebeli versiyonu elde edilmiştir. Çalışılan sistem sınır koşullarıyla Sturm Liouville operatörünün uyumlu türevli daha genel bir formatı ispatlanmıştır. Ayrıca, gözününe alınan bu problem için özdeğerlerin reeliği ve özfonksiyonların $\boldsymbol{\alpha}$ - ortoganalliğini ispatlamıştır. İlaveten modifiye edilmiş Coloumb Potansiyele sahip Sturm Liouville problemin çözümünün görüntüsü bulunmuştur. Sonuçlar grafiklerle karş̧laştırmalı olarak gösterilmiştir.

Anahtar Kelimeler-Conformable (uyumlu) Türev, Özdeğer, Özfonksiyonlar, Spektral, Coulomb Potansiyeli

\footnotetext{
1*Sorumlu yazar iletişim: erdalmat@yahoo.com (https://orcid.org/0000-0002-2275-8061)

Matematik Bölümü, Fırat Üniversitesi, Elazığ, Türkiye

2İletişim: isammath94@gmail.com (https://orcid.org/0000-0003-1344-9977)

University of Zakho, Iraq
} 


\title{
Conformable Sturm-Liouville Problem with Modified Coulomb Potential
}

\begin{abstract}
In this paper, Conformable derivative order version of the Sturm-Liouville problem having modified Coulomb potential is obtained. The studied system proves the shape of the conformable derivative general statement of the Sturm-Liouville operator with boundary conditions. Furthermore, real of eigenvalues and $\boldsymbol{\alpha}$-orthogonal of eigenfunctions have been proved for the problem considered. Additionally, the representation of the solution of the Sturm-Liouville problem having modified Coulomb potential is found. The results are shown comparatively by figures.
\end{abstract}




\section{INTRODUCTION}

The idea of the fractional computation is as old as usual calculus. This concept was made when L'Hospital in 1695 asked specifically what does the means of $\frac{d^{n} f}{d x^{n}}$ where $n=\frac{1}{2}$. From that time on, intensive research of fractional calculus in the last and current centuries has performed many types of research [1-4]. Some researchers like Fourier, Liouville, Weyl, Riemann, Abel, Leibniz, Grünwald, and Letnikov attempted to put a definition of the fractional derivative. Some of them used an integral form for the fractional derivative. From these outcomes, the most well-known notions of the fractional derivative are Riemann-Liouville definition also Caputo definition[5-8].

I. Riemann-Liouville definition. Assume that $0<\alpha \leq 1$. Then the derivative of the function $f$ with order $\alpha$ is defined by

$$
D_{a}^{\alpha}(f)(t)=\frac{1}{\Gamma(n-\alpha)} \frac{d^{n}}{d t^{n}}\left[\int_{a}^{t} \frac{f(t)}{(t-x)^{\alpha-n+1}} d x\right] .
$$

II. Caputo definition. The derivative of the function $f$ with order $\alpha$ for $\alpha \epsilon[n-1, n)$ is defined as follows

$$
D_{a}^{\alpha}(f)(t)=\frac{1}{\Gamma(n-\alpha)}\left[\int_{a}^{t} \frac{f^{(n)}(t)}{(t-x)^{\alpha-n+1}} d x\right]
$$

Where $D_{a}^{\alpha}$ is an operator of the left fractional derivative and $\frac{d^{n}}{d t^{n}}$ is an operator of the derivative with integer order, the acquired fractional derivatives in this measurement appeared difficult also do not satisfy some of the main properties the same as normal derivatives for example chain rule, the product rule, and so on. The concept Riemann-Liouville derivative does not fulfill $D_{a}^{\alpha}(1)=0,\left({ }^{c} D_{a}^{\alpha}(1)=0\right.$ for the Caputo definition), where $\alpha$ does not belong to a natural number. However, some properties of these fragmentary administrators carry on well at times[9-10]. In recent times, Khalil et al. give a new-well definition of local derivative named conformable derivative which is applied a limit form same ordinary derivative form and satisfies all previous properties. Additionally, they define a conformable integral with order $\alpha \epsilon(0,1]$. They also really evaluated and showed conformable Rolle's theorem and mean value theorem through definition that founded by them [11]-[14].

\section{BASIC DEFINITION AND THEOREM}

Definition 2.1. [11]Assume that $f:[0, \infty) \rightarrow \mathbb{R}$ be a function, then the definition of the left conformable derivative of the function $f$ with order $\alpha$, where $0<\alpha \leq 1$ is defined as follows

$$
\left(T_{\alpha}^{a} f\right)(t)=\lim _{\varepsilon \rightarrow 0} \frac{f\left(t+\varepsilon(t-a)^{1-\alpha}\right)-f(t)}{\varepsilon},
$$

for every $t>a$. write $T_{\alpha}$, where $a=0$. If $\left(T_{\alpha}^{a} f\right)(t)$ exists on $(a, b)$, then we have

$$
\left(T_{\alpha}^{a} f\right)(a)=\lim _{t \rightarrow a^{+}}\left(T_{\alpha}^{a} f\right)(t)
$$

In [15] let us $f$ be a differentiable function on the interval $(a, b)$ and $\alpha \in(0,1]$, so that

$$
\left(T_{\alpha}^{a} f\right)(t)=(t-a)^{1-\alpha} f^{\prime}(x) .
$$


Definition 2.2. [11]The right conformable derivative of $f:[0, \infty) \rightarrow \mathbb{R}$ with order $\alpha, \alpha \in(0,1]$ is defined as follows

$$
\left({ }_{\alpha}^{b} T f\right)(t)=\lim _{\varepsilon \rightarrow 0} \frac{f\left(t+\varepsilon(b-t)^{1-\alpha}\right)-f(t)}{\varepsilon},
$$

for every $t<b$. We write ${ }_{\alpha} T$, where $b=0$. If $\left({ }_{\alpha}^{b} T f\right)(t)$ exists on $(a, b)$, then we have

$$
\left({ }_{\alpha}^{b} T f\right)(b)=\lim _{t \rightarrow b^{-}}\left({ }_{\alpha}^{b} T f\right)(t)
$$

In [15] if $f$ is differentiable function on the interval $(a, b)$, then we can define the right conformable derivative of $0<\alpha \leq 1$ as

$$
\left({ }_{\alpha}^{b} T f\right)(t)=-(b-t)^{1-\alpha} f^{\prime}(t)
$$

If the conformable derivative of the function $f$ exists, then the given function $f$ is said to be $\alpha$ differentiable [16].

Definition 2.3.[16]Suppose that $\alpha \epsilon(n, n+1]$ for $n \in \mathbb{N}$, and $f$ be $\mathrm{n}$-differentiable function at a pointt for $t>0$, the conformable derivative with order $\alpha$ of $f$ is defined by

$$
\left(T_{\alpha} f\right)(t)=\lim _{\varepsilon \rightarrow 0} \frac{f^{([\alpha]-1)}\left(t+\varepsilon t^{[\alpha]-\alpha}\right)-f^{([\alpha]-1)}(t)}{\varepsilon} .
$$

Where $\lceil\alpha\rceil$ be the smallest integer number that greater or equal to $\alpha$.Besides, for $\alpha \epsilon(n, n+1], n$ is a positive integer we can say that

$$
\left(T_{\alpha} f\right)(t)=t^{([\alpha]-\alpha)} f^{[\alpha]}(t) .
$$

From the above definition, we can say $f$ is $(n+1)$-differentiable function at non-negative number $t$.

Definition 2.4.[15]Assume that $f:(0, \infty) \rightarrow \mathbb{R}$ be a given function and $\alpha \in(0,1]$. so that, the definition of left conformable integral of $f$ with order $\alpha$ is given by

$$
\begin{aligned}
& \left(I_{\alpha}^{a} f\right)(t)=\int_{a}^{t} f(x) d_{\alpha}(x, a) \\
& =\int_{a}^{t}(x-a)^{a-1} f(x) d x .
\end{aligned}
$$

We are writing $I_{\alpha}$ and $d_{\alpha} x$ where $a=0$.

Definition 2.5.[15] The right conformable integral with order $\alpha$ of the function $f$ where $f:(0, \infty) \rightarrow \mathbb{R}$ and $\alpha \in(0,1]$ is defined by 


$$
\begin{gathered}
\left({ }_{\alpha}^{b} I f\right)(t)=\int_{t}^{b} f(x) d_{\alpha}(b, x) \\
=\int_{t}^{b}(b-x)^{a-1} f(x) d x .
\end{gathered}
$$

If $b=0$, then we write ${ }_{\alpha} I$ and $d_{\alpha} x$.

Definition 2.6. [15] Let $f$ be a given function and $\beta=\alpha-n$ where $0<\alpha \leq 1$, then the left conformable integral definition that starting from $a>0$ of $f$ is

$$
\left(I_{\alpha}^{a} f\right)(t)=\boldsymbol{I}_{n+1}^{a}\left((t-a)^{\beta-1} f\right)=\frac{1}{n !}\left[\int_{a}^{t}(t-x)^{n}(x-a)^{\beta-1} f(x) d x\right] .
$$

Perceive that if $\alpha=n+1$, then we obtain $\beta=1$. Therefore, by way of Cauchy formula, the iterative integral of $f$ over $n+1$ times on the interval $(a, t]$ is provided

$$
\left(I_{\alpha}^{a} f\right)(t)=\left(\mathbf{I}_{n+1}^{a} f\right)(t)=\frac{1}{n !}\left[\int_{a}^{t}(t-x)^{n} f(x) d x\right]
$$

Theorem 2.7.[15] Assume that $\alpha \in(0,1]$ and $f:(0, \infty) \rightarrow \mathbb{R}$ be given continuous function, for every $t>a$

$$
T_{\alpha}^{a} I_{\alpha}^{a} f(t)=f(t)
$$

And

$$
{ }_{\alpha}^{b} T_{\alpha}^{b} I f(t)=f(t)
$$

If $f^{n}(t)$ is continuous, then for $\alpha \in(n, n+1]$ both equations (15) and (16) hold.

Lemma 2.8. [15]Assume $0<\alpha \leq 1$ and $f:[a, b) \rightarrow \mathbb{R}$ be a differentiable function, then for all $t>0$

$$
I_{\alpha}^{a} T_{\alpha}^{a} f(t)=f(t)-f(a) .
$$

And

$$
{ }_{\alpha}^{b} I_{\alpha}^{b} T(f)(t)=f(t)-f(b)
$$

Theorem 2.9. [15](Conformable integration by parts). Assume that $f$ and $g$ be two given differentiable functions such that $f, g:[a, b] \rightarrow \mathbb{R}$, then 


$$
\int_{a}^{b} f(x) T_{\alpha}^{a}(g)(x) d_{\alpha}(x, a)=\left.f g\right|_{a} ^{b}-\int_{a}^{b} g(x) T_{\alpha}^{a}(f)(x) d_{\alpha}(x, a)
$$

Theorem 2.10.[17]Let $p$ be a point in real number, then for $p \geq 1$ the set $L_{\alpha}^{p}([a, b], \mathbb{R}),(a \geq 0)$ be a Banach space along with the norm such that defined for $f \in L_{\alpha}^{p}([a, b], \mathbb{R})$ as

$$
\|f\|_{L_{\alpha}^{p}([a, b], \mathbb{R})}=\left(\int_{a}^{b}|f(t)|^{p} d_{\alpha} t\right)^{1 / p}
$$

Furthermore, space $L_{\alpha}^{2}([a, b], \mathbb{R})$ is a Hilbert space along with inner product provided for all $(f, g) \in$ $L_{\alpha}^{p}([a, b], \mathbb{R}) \times L_{\alpha}^{p}([a, b], \mathbb{R})$

$$
\langle f, g\rangle_{L_{\alpha}^{2}([a, b], \mathbb{R})}=\int_{a}^{b} f(t) g(t) d_{\alpha} t
$$

\section{MAIN RESULTS}

For $a<x<b$, we are looking at the conformable extension thorough account of the Sturm-Liouville eigenvalue problem having modified Coulomb potential

$$
-T_{\alpha}^{a} T_{\alpha}^{a} y+\alpha^{2}\left(\frac{A}{x^{\alpha}}+q(x)\right) y=\alpha^{2} \lambda y .
$$

where $\frac{y(x)}{x^{\alpha}} \in C^{2 \alpha}[0, \pi]$. We analyze (22) with boundary conditions

$$
\begin{array}{ll}
c_{1} y(0)+c_{2} y^{\prime}(0)=0, & c_{1}{ }^{2}+c_{2}{ }^{2}>0 \\
r_{1} y(\pi)+r_{2} y^{\prime}(\pi)=0, & r_{1}{ }^{2}+r_{2}{ }^{2}>0 .
\end{array}
$$

If $T_{\alpha}^{a} T_{\alpha}^{a} y$ is continuous on $[a . b]$ then we conclude that $y$ is $2 \alpha$-continuously differentiable. Also, $y \in$ $C^{2 \alpha}[a, b]$ wherey $\in C^{1}[a, b]$ and be $2 \alpha$ - continuously differentiable on $[a, b]$.

Suppose that $L$ be a linear operator described on certain elements such that

$$
L(y, \alpha)=-T_{\alpha}^{a} T_{\alpha}^{a} y+\alpha^{2}\left(\frac{A}{x^{\alpha}}+q(x)\right) y,
$$

then we can write (22) as

$$
L(y, \alpha)=\alpha^{2} \lambda y
$$

In this process, we generalize the easy outcome of a well-known Lagrange identity.

Theorem 3.1. Let $y_{1}, y_{2}$ be $2 \alpha$-continuously differentiable functions on the interval $[0, \lambda]$, so that the following equation maintain true 
$\int_{0}^{\pi}\left(y_{2} L\left(y_{1}, \alpha\right)-y_{1} L\left(y_{2}, \alpha\right)\right) d_{\alpha}(x)=\left.\left(y_{2} T_{\alpha}^{a} y_{1}-y_{1} T_{\alpha}^{a} y_{2}\right)\right|_{0} ^{\pi}$.

This theorem is said to be the conformable Lagrange identity.

Proof. From (22), we see that

$$
\begin{gathered}
y_{2} L\left(y_{1}, \alpha\right)-y_{1} L\left(y_{2}, \alpha\right)=-y_{2} T_{\alpha}^{a} T_{\alpha}^{a} y_{1}+\alpha^{2}\left(\frac{A}{x^{\alpha}}+q(x)\right) y_{1} y_{2} \\
+y_{1} T_{\alpha}^{a} T_{\alpha}^{a} y_{2}-\alpha^{2}\left(\frac{A}{x^{\alpha}}+q(x)\right) y_{1} y_{2} \\
=y_{1} T_{\alpha}^{a} T_{\alpha}^{a} y_{2}-y_{2} T_{\alpha}^{a} T_{\alpha}^{a} y_{1}
\end{gathered}
$$

by using conformable integration with order $\alpha$ of (27) and using conformable integration by parts from Theorem 3.1 , we obtain

$$
\begin{aligned}
& \int_{0}^{\pi}\left(y_{2} L\left(y_{1}, \alpha\right)-y_{1} L\left(y_{2}, \alpha\right)\right) d_{\alpha}(x) \\
& =\int_{0}^{\pi} y_{1} T_{\alpha}^{a} T_{\alpha}^{a} y_{2} d_{\alpha}(x)-\int_{0}^{\pi} y_{2} T_{\alpha}^{a} T_{\alpha}^{a} y_{1} d_{\alpha}(x) \\
& =\left.y_{1} T_{\alpha}^{a} y_{2}\right|_{0} ^{\pi}-\int_{0}^{\pi} T_{\alpha}^{a} y_{1} T_{\alpha}^{a} y_{2} d_{\alpha}(x) \\
& \quad-\left.y_{2} T_{\alpha}^{a} y_{1}\right|_{0} ^{\pi}+\int_{0}^{\pi} T_{\alpha}^{a} y_{1} T_{\alpha}^{a} y_{2} d_{\alpha}(x) \\
& =\left.\left(y_{1} T_{\alpha}^{a} y_{2}-y_{2} T_{\alpha}^{a} y_{1}\right)\right|_{0} ^{\pi} .
\end{aligned}
$$

Proposition 3.2. Assume that $y_{1}$ and $y_{2}$ be two given function in $C^{1}[0, \lambda]$ such that satisfy the boundary conditions (23), then the following holds true

$$
\left.\left(y_{2} T_{\alpha}^{a} y_{1}-y_{1} T_{\alpha}^{a} y_{2}\right)\right|_{0} ^{\pi}=0 .
$$

Proof: In (29) we have

$$
\begin{aligned}
\left.\left(y_{2} T_{\alpha}^{a} y_{1}-y_{1} T_{\alpha}^{a} y_{2}\right)\right|_{0} ^{\pi}= & y_{2}(\pi)\left(T_{\alpha}^{a} y_{1}\right)(\pi)-y_{1}(\pi)\left(T_{\alpha}^{a} y_{2}\right)(\pi) \\
& -\left(y_{2}(0)\left(T_{\alpha}^{a} y_{1}\right)(0)+y_{1}(0)\left(T_{\alpha}^{a} y_{2}\right)(0)\right) .
\end{aligned}
$$

Since $c_{1}{ }^{2}+c_{2}{ }^{2}>0$, and $r_{1}{ }^{2}+r_{2}{ }^{2}>0$, in the beginning, we assume that $c_{1} \neq 0$ and $r_{1} \neq 0$, without loss of simplification and the proof of other cases going to be gotten similarly. Now in (23), we get 


$$
\begin{aligned}
& y(0)=-\frac{c_{2}}{c_{1}} y^{\prime}(0), \\
& y(\pi)=-\frac{r_{2}}{r_{1}} y^{\prime}(\pi) .
\end{aligned}
$$

By the above definitions $\left(T_{\alpha}^{a} y_{1}\right)(x)=(x-a)^{1-\alpha} y^{\prime}{ }_{1}(x)$ and $\left(T_{\alpha}^{a} y_{2}\right)(x)=(x-a)^{1-\alpha} y^{\prime}{ }_{2}(x)$ because $y_{1}, y_{2} \in$ $C^{1}[a, b]$. Now by applying that and (29) in equation (28), we get

$$
\begin{aligned}
& \left(y_{2}(\pi)\left(T_{\alpha}^{a} y_{1}\right)(\pi)-y_{1}(\pi)\left(T_{\alpha}^{a} y_{2}\right)(\pi)\right) \\
& =-\frac{r_{2}}{r_{1}} y_{2}^{\prime}{ }_{2}(\pi)\left(T_{\alpha}^{a} y_{1}\right)(\pi)+\frac{r_{2}}{r_{1}} y_{1}^{\prime}(\pi)\left(T_{\alpha}^{a} y_{2}\right)(\pi) \\
& \quad=-\frac{r_{2}}{r_{1}}\left(y^{\prime}{ }_{2}(\pi)(\pi-a)^{1-\alpha} y_{1}^{\prime}{ }_{1}(\pi)-y_{1}^{\prime}(\pi)(\pi-a)^{1-\alpha} y^{\prime}{ }_{2}(\pi)\right)
\end{aligned}
$$

$=0$.

Similarly

$$
y_{2}(0)\left(T_{\alpha}^{a} y_{1}\right)(0)+y_{1}(0)\left(T_{\alpha}^{a} y_{2}\right)(0)=0 .
$$

Therefore the proof has been demonstrated.

Definition 3.3. [8] Let $f$ and $g$ be two given function then we say $f$ and $g$ are $\alpha$-orthogonal in relation to the weight function $\mathcal{U}(t) \geq 0$, where

$$
\int_{0}^{\pi} u(x) f(x) g(x) d_{\alpha}(x)=0
$$

Theorem 3.4.Both eigenfunctions $y_{1}$ and $y_{2}$ of the Sturm-Liouville eigenvalue problem (22) - (23) corresponding to different eigenvalues $\lambda_{1}$ and $\lambda_{2}$ respectively are $\alpha$-orthogonal where $\left(y_{1}, y_{2}\right) \in L_{\alpha}^{2}([0, \pi], \mathbb{R}) \times$ $L_{\alpha}^{2}([0, \pi], \mathbb{R})$.

Proof. in (25) we see that

$$
\begin{aligned}
& L\left(y_{1}, \alpha\right)=\alpha^{2} \lambda_{1} y_{1} \\
& L\left(y_{2}, \alpha\right)=\alpha^{2} \lambda_{2} y_{2}
\end{aligned}
$$

multiplying (35) by $y_{2}$ also, multiplying (36) by $y_{1}$ and subtracting both equations, then we receive that

$$
\left(\lambda_{1}-\lambda_{2}\right) \alpha^{2} y_{1} y_{2}=y_{2} L\left(y_{1}, \alpha\right)-y_{1} L\left(y_{2}, \alpha\right)
$$


By using the conformable integration with order $\alpha$ and the conformable Lagrange identity theorem, we see that

$$
\begin{aligned}
& \left(\lambda_{1}-\lambda_{2}\right) \alpha^{2} \int_{0}^{\pi} y_{1} y_{2} d_{\alpha}(x)=\int_{0}^{\pi}\left(y_{2} L\left(y_{1}, \alpha\right)-y_{1} L\left(y_{2}, \alpha\right)\right) d_{\alpha}(x) \\
& =\left.\left[y_{2} T_{\alpha}^{a} y_{1}-y_{1} T_{\alpha}^{a} y_{2}\right]\right|_{0} ^{\pi} \\
& =0,
\end{aligned}
$$

it is according to Proposition 3.2. In (38) we get $\int_{0}^{\pi} y_{1} y_{2} d_{\alpha}(x)=0$ because the eigenvalues $\lambda_{1}$ and $\lambda_{2}$ are distinct and $\alpha^{2} \neq 0$, this fulfills the proof.

Theorem 3.5. Eigenvalues of the conformable Sturm-Liouville eigenvalue problem (21) - (23) are real.

Proof. Assume that $y$ be a solution of the conformable Sturm-Liouville eigenvalue problem (21) (23). By applying the complex conjugate of (21) - (23), then we obtain that

$$
\begin{aligned}
& L(\bar{y}, \alpha)=-T_{\alpha}^{a} T_{\alpha}^{a} \bar{y}+\alpha^{2}\left(\frac{A}{x^{\alpha}}+q(x)\right) \bar{y} \\
& =\alpha^{2} \lambda \bar{y} . \\
& c_{1} \bar{y}(0)+c_{2} \bar{y}^{\prime}(0)=0, \quad c_{1}{ }^{2}+c_{2}^{2}>0 . \\
& r_{1} \bar{y}(\pi)+r_{2} \bar{y}^{\prime}(\pi)=0, \quad r_{1}{ }^{2}+r_{2}{ }^{2}>0 .
\end{aligned}
$$

By using comparable measures to the proof of Theorem 3.4 for $y_{1}=y, y_{2}=\bar{y}, \lambda_{1}=\lambda$ and $\lambda_{2}=\bar{\lambda}$, we get

$$
\begin{aligned}
& \alpha^{2}(\lambda-\bar{\lambda}) \int_{0}^{\pi}|y(x)|^{2} d_{\alpha}(x)=\int_{0}^{\pi}(\bar{y} L(y, \alpha)-y L(\bar{y}, \alpha)) d_{\alpha}(x) \\
& =\left.\left[\bar{y} T_{\alpha}^{a} y-y \overline{T_{\alpha}^{a} y_{2}}\right]\right|_{0} ^{\pi} \\
& =0
\end{aligned}
$$

So, $\int_{0}^{\pi}|y(x)|^{2} d_{\alpha}(x) \neq 0$ because $|y(x)|^{2}$ is positive, since $\alpha^{2}>0$, Then $\lambda-\bar{\lambda}=0 \Rightarrow \lambda=\bar{\lambda}$. Hence, the eigenvalues must be real.

Definition 3.6.[7] We assume that $y_{1}(t), y_{2}(t), \cdots, y_{n}(t)$ are $(n-1)$ times $\alpha$-differentiable functions and $0<\alpha \leq 1$. So that, we are denoting the conformable Wronskian of that functions by $W_{\alpha}\left(y_{1}, y_{2}, \cdots, y_{n}\right)$ and defined as

$$
W_{\alpha}\left(y_{1}, y_{2}, \ldots, y_{n}\right)=\left|\begin{array}{cccr}
y_{1} & y_{2} & \ldots & y_{n} \\
T_{\alpha}^{a} y_{1} & T_{\alpha}^{a} y_{2} & \ldots & T_{\alpha}^{a} y_{n} \\
\vdots & \vdots & \ldots & \vdots \\
{ }^{(n-1)} T_{\alpha}^{a} y_{1} & { }^{(n-1)} T_{\alpha}^{a} y_{2} & \ldots & { }^{(n-1)} T_{\alpha}^{a} y_{n}
\end{array}\right|
$$


Theorem 3.7. Suppose that $y \in C^{2 \alpha}[0, \pi]$, then the representation of the solution of the Sturm-Liouville eigenvalue problem having modified Coulomb potential

$$
-T_{\alpha} T_{\alpha} y+\alpha^{2}\left(\frac{A}{x^{\alpha}}+q(x)\right) y=\alpha^{2} \lambda y
$$

With both initial conditions $y(0, \lambda)=1$ and $T_{\alpha}^{0} y(0, \lambda)=h$ is

$$
y(x)=\cos \left(\sqrt{\lambda} x^{\alpha}\right)+\frac{h}{\alpha \sqrt{\lambda}} \sin \left(\sqrt{\lambda} x^{\alpha}\right)+\frac{\alpha}{\sqrt{\lambda}} \int_{0}^{x}\left(\frac{A}{t^{\alpha}}+q(t)\right) y(t) \sin \left(\sqrt{\lambda}\left(x^{\alpha}-t^{\alpha}\right)\right) d_{\alpha} t
$$

Proof. To get a solution of (43) we need to find $y_{h}$ and $y_{p}$.

$$
T_{\alpha} T_{\alpha} y+\alpha^{2} \lambda y=0
$$

we look for $y=e^{r \frac{x^{\alpha}}{\alpha}}$, then $T_{\alpha}{ }_{\alpha} y=r e^{r \frac{x^{\alpha}}{\alpha}}$ and $T_{\alpha} T_{\alpha} y=r^{2} e^{r \frac{x^{\alpha}}{\alpha}}$. So, in (45) we get $\left(r^{2}+\alpha^{2} \lambda\right) e^{r \frac{x^{\alpha}}{\alpha}}=0 \Rightarrow$. Hence, we obtain that $r_{1}=\alpha \sqrt{\lambda} i$ and $r_{2}=-\alpha \sqrt{\lambda} i$. So,

$$
y_{h}=c_{1} \cos \left(\sqrt{\lambda} x^{\alpha}\right)+c_{2} \sin \left(\sqrt{\lambda} x^{\alpha}\right)
$$

Suppose that $y_{1}=\cos \left(\sqrt{\lambda} x^{\alpha}\right)$ and $y_{2}=\sin \left(\sqrt{\lambda} x^{\alpha}\right)$, then we have

$$
\begin{aligned}
& y_{p}=u_{1} y_{1}+u_{2} y_{2} \\
& T_{\alpha} y_{p}=T_{\alpha} u_{1} y_{1}+u_{1} T_{\alpha} y_{1}+T_{\alpha} u_{2} y_{2}+u_{2} T_{\alpha} y_{2} \\
& T_{\alpha} u_{1} y_{1}+T_{\alpha} u_{2} y_{2}=0 \\
& T_{\alpha} T_{\alpha} y_{p}=T_{\alpha} u_{1} T_{\alpha} y_{1}+u_{1} T_{\alpha} T_{\alpha} y_{1}+T_{\alpha} u_{2} T_{\alpha} y_{2}+u_{2} T_{\alpha} T_{\alpha} y_{2} .
\end{aligned}
$$

By taking (47) and (50) in (43), we get

$$
\begin{aligned}
& -T_{\alpha} u_{1} T_{\alpha} y_{1}-u_{1} T_{\alpha} T_{\alpha} y_{1}-T_{\alpha} u_{2} T_{\alpha} y_{2}-u_{2} T_{\alpha} T_{\alpha} y_{2}+\alpha^{2}\left(\frac{A}{x^{\alpha}}+q(x)\right) y=\alpha^{2} \lambda u_{1} y_{1}+\alpha^{2} \lambda u_{2} y_{2} \\
& T_{\alpha} u_{1} T_{\alpha} y_{1}+T_{\alpha} u_{2} T_{\alpha} y_{2}+u_{1}\left(T_{\alpha} T_{\alpha} y_{1}+\alpha^{2} \lambda y_{1}\right)+u_{2}\left(T_{\alpha} T_{\alpha} y_{2}+\alpha^{2} \lambda y_{2}\right)=\alpha^{2}\left(\frac{A}{x^{\alpha}}+q(x)\right) y \\
& T_{\alpha} u_{1} T_{\alpha} y_{1}+T_{\alpha} u_{2} T_{\alpha} y_{2}=\alpha^{2}\left(\frac{A}{x^{\alpha}}+q(x)\right) y
\end{aligned}
$$

So, by (49) and (51) we receive that 


$$
\begin{aligned}
& T_{\alpha} u_{1} \cos \left(\sqrt{\lambda} x^{\alpha}\right)+T_{\alpha} u_{2} \sin \left(\sqrt{\lambda} x^{\alpha}\right)=0 \\
& -\alpha \sqrt{\lambda} T_{\alpha} u_{1} \sin \left(\sqrt{\lambda} x^{\alpha}\right)+\alpha \sqrt{\lambda} T_{\alpha} u_{1} \cos \left(\sqrt{\lambda} x^{\alpha}\right)=\alpha^{2}\left(\frac{A}{x^{\alpha}}+q(x)\right) y
\end{aligned}
$$

By applying Definition 3.6 to find $\alpha$-Wronskian of $y_{1}$ and $y_{2}$ as

$$
\begin{aligned}
& W_{\alpha}\left(y_{1}, y_{2}\right)=y_{1} T_{\alpha} y_{2}-y_{2} T_{\alpha} y_{1} \\
& =\alpha \sqrt{\lambda}\left(\cos \left(\sqrt{\lambda} x^{\alpha}\right)\right)^{2}+\alpha \sqrt{\lambda}\left(\sin \left(\sqrt{\lambda} x^{\alpha}\right)\right)^{2} \\
& =\alpha \sqrt{\lambda} \\
& T_{\alpha} u_{1}=\frac{\left|\begin{array}{cr}
0 & \sin \left(\sqrt{\lambda} x^{\alpha}\right) \\
\alpha^{2}\left(\frac{A}{x^{\alpha}}+q(x)\right) y & \cos \left(\sqrt{\lambda} x^{\alpha}\right)
\end{array}\right|}{\alpha \sqrt{\lambda}}=-\frac{\alpha}{\sqrt{\lambda}}\left(\frac{A}{x^{\alpha}}+q(x)\right) y \sin \left(\sqrt{\lambda} x^{\alpha}\right) . \\
& T_{\alpha} u_{2}=\frac{\left|\begin{array}{ll}
\cos \left(\sqrt{\lambda} x^{\alpha}\right) & 0 \\
\sin \left(\sqrt{\lambda} x^{\alpha}\right) & \alpha^{2}\left(\frac{A}{x^{\alpha}}+q(x)\right) y
\end{array}\right|}{\alpha \sqrt{\lambda}}=\frac{\alpha}{\sqrt{\lambda}}\left(\frac{A}{x^{\alpha}}+q(x)\right) y \cos \left(\sqrt{\lambda} x^{\alpha}\right) . \\
& u_{1}=-\frac{\alpha}{\sqrt{\lambda} \int_{0}^{x}\left(\frac{A}{t^{\alpha}}+q(t)\right) y(t) \sin \left(\sqrt{\lambda} t^{\alpha}\right) d_{\alpha} t .} \\
& u_{2}=\frac{\alpha}{\sqrt{\lambda}} \int_{0}^{x}\left(\frac{A}{t^{\alpha}}+q(t)\right) y(t) \cos \left(\sqrt{\lambda} t^{\alpha}\right) d_{\alpha} t .
\end{aligned}
$$

By taking the value of $u_{1}$ and $u_{2}$ in (47) we obtain

$$
\begin{gathered}
y_{p}=-\frac{\alpha \cos \left(\sqrt{\lambda} x^{\alpha}\right)}{\sqrt{\lambda}} \int_{0}^{x}\left(\frac{A}{t^{\alpha}}+q(t)\right) y(t) \sin \left(\sqrt{\lambda} t^{\alpha}\right) d_{\alpha} t \\
+\frac{\alpha \sin \left(\sqrt{\lambda} x^{\alpha}\right)}{\sqrt{\lambda}} \int_{0}^{x}\left(\frac{A}{t^{\alpha}}+q(t)\right) y(t) \cos \left(\sqrt{\lambda} t^{\alpha}\right) d_{\alpha} t \\
=\frac{\alpha}{\sqrt{\lambda}} \int_{0}^{x}\left(\frac{A}{t^{\alpha}}+q(t)\right) y(t)\left[\sin \left(\sqrt{\lambda} x^{\alpha}\right) \cos \left(\sqrt{\lambda} t^{\alpha}\right)-\cos \left(\sqrt{\lambda} x^{\alpha}\right) \sin \left(\sqrt{\lambda} t^{\alpha}\right)\right] d_{\alpha} t \\
=\frac{\alpha}{\sqrt{\lambda}} \int_{0}^{x}\left(\frac{A}{t^{\alpha}}+q(t)\right) y(t) \sin \left(\sqrt{\lambda}\left(x^{\alpha}-t^{\alpha}\right)\right) d_{\alpha} t .
\end{gathered}
$$


Thus, from (46) and (59) we have

$$
y(x)=c_{1} \cos \left(\sqrt{\lambda} x^{\alpha}\right)+c_{2} \sin \left(\sqrt{\lambda} x^{\alpha}\right)+\frac{\alpha}{\sqrt{\lambda}} \int_{0}^{x}\left(\frac{A}{t^{\alpha}}+q(t)\right) y(t) \sin \left(\sqrt{\lambda}\left(x^{\alpha}-t^{\alpha}\right)\right) d_{\alpha} t .
$$

By applying the boundary conditions, we get that $1=c_{1} \cos 0+c_{2} \sin 0 \Rightarrow c_{1}=1$ and $h=-\alpha \sqrt{\lambda} c_{1} \sin 0+$ $\alpha \sqrt{\lambda} c_{2} \cos 0 \Rightarrow c_{2}=\frac{h}{\alpha \sqrt{\lambda}}$, then in (60), we get

$$
y(x)=\cos \left(\sqrt{\lambda} x^{\alpha}\right)+\frac{h}{\alpha \sqrt{\lambda}} \sin \left(\sqrt{\lambda} x^{\alpha}\right)+\frac{\alpha}{\sqrt{\lambda}} \int_{0}^{x}\left(\frac{A}{t^{\alpha}}+q(t)\right) y(t) \sin \left(\sqrt{\lambda}\left(x^{\alpha}-t^{\alpha}\right)\right) d_{\alpha} t .
$$

Application 3.8. In [18] for $0<\alpha \leq 1$ by using the Frobenius method we obtained the solution of modified power series within a regular singular point $x=0$ of

$$
-T_{\alpha} T_{\alpha} y+\alpha^{2} \frac{1}{x^{\alpha}} y=0
$$

as

$$
y(x)=\sum_{n=0}^{\infty} \frac{c_{0}}{n !(n+1) !} x^{(n+1) \alpha}
$$

Equation (61) is the first solution of conformable Sturm-Liouville eigenvalue problem having modified Coulomb potential in the homogeneous case for $A$

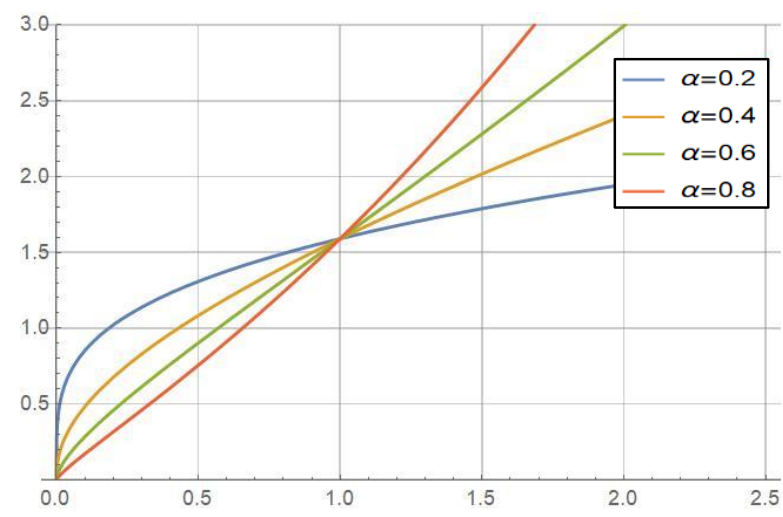

Figure. 1. The solution of the equation (62) when $\operatorname{in}(63) c_{0}=1$. 


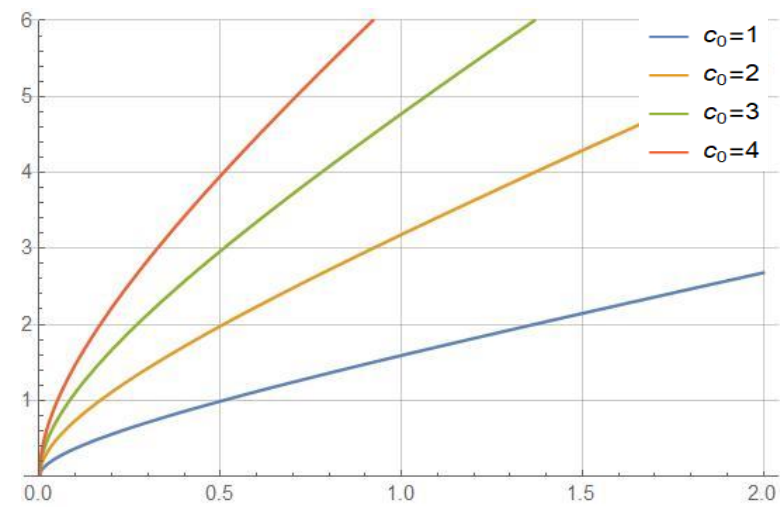

Figure 2. The solution of the equation (62) when in (63) $\alpha=0.5$.

Furthermore, the first solution to

$$
T_{\alpha} T_{\alpha} y-\alpha^{2} \frac{1}{x^{\alpha}} y=\alpha^{2} \lambda y
$$

where $0<\alpha \leq 1$ is

$$
y(x)=c_{0}\left(x^{\alpha}+\frac{1}{2} x^{2 \alpha}+\frac{(2 \lambda+1)}{2.2 .3} x^{3 \alpha}+\frac{(8 \lambda+1)}{2 \cdot 2 \cdot 3 \cdot 3.4} x^{4 \alpha}+\frac{24 \lambda^{2}+(20 \lambda+1)}{2 \cdot 2 \cdot 3 \cdot 3 \cdot 4.4 .5} x^{5 \alpha}+\cdots\right) .
$$

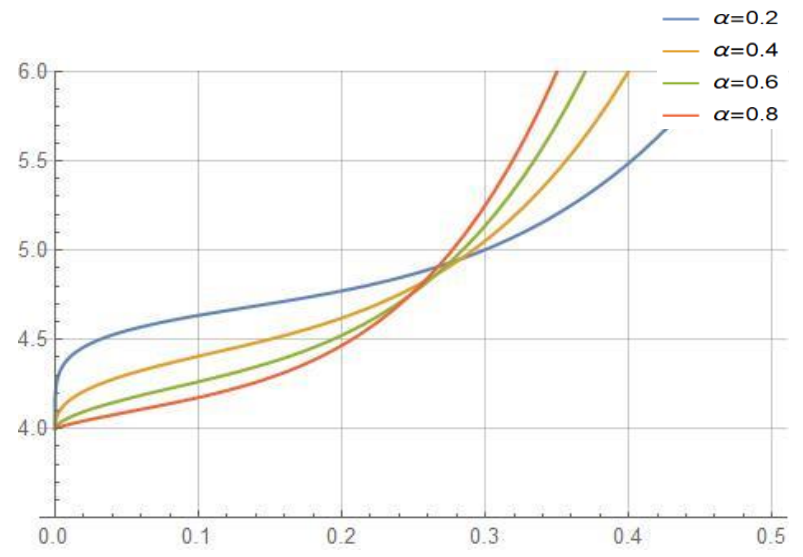

Figure 3. The solution of the equation (64) when in $(65) \lambda=1, c_{0}=1$. 


\begin{tabular}{|c|c|c|}
\hline & $\begin{array}{l}\text { BŞEÜ Fen Bilimleri Dergisi } \\
7(2), 698-713,2020\end{array}$ & $\begin{array}{r}\text { BSEU Journal of Science } \\
\text { DOI: } 10.35193 / \text { bseufbd. } 668272\end{array}$ \\
\hline on & & 58-7575 (http://dergipark.gov.tr/bseufbd) \\
\hline
\end{tabular}

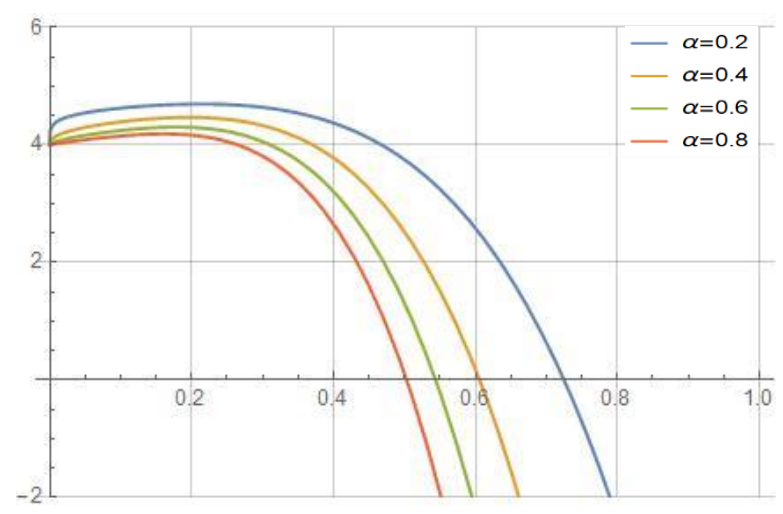

Figure 4. The solution of the equation (64) when in (65) $c_{0}=1, \lambda=-1$.

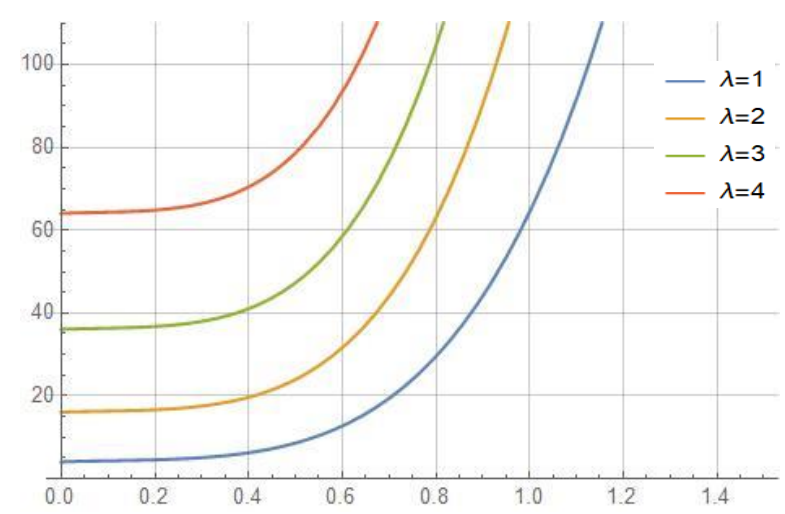

Figure 5. The solution of the equation (64) when in (65) $c_{0}=1, \alpha=0.5$.

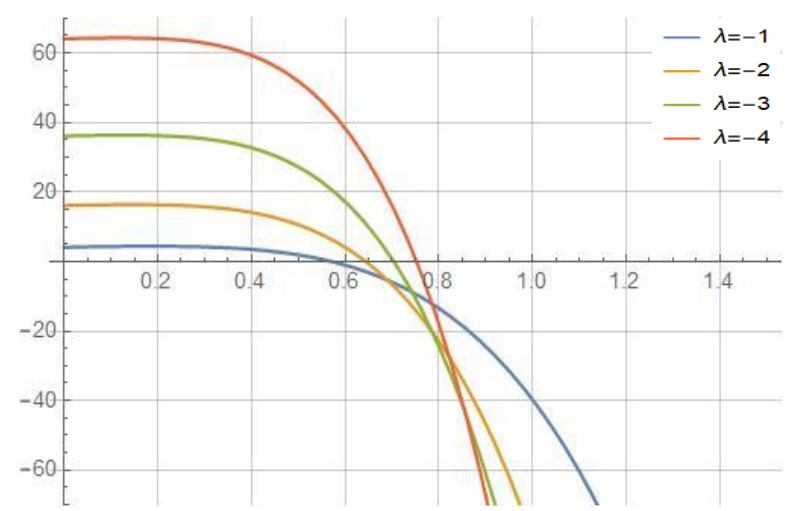

Figure 6. The solution of the equation (64) when in (65) $\alpha=0.5, c_{0}=1$.

\section{CONCLUSION}

The conformable extension through the study of the Sturm-Liouville eigenvalue problem having modified Coulomb potential is recognized. We proved the conformable Lagrange identity theorem by using conformable integration by parts. Defined orthogonality of two functions and using this definition can show that the eigenfunctions of the Sturm-Liouville eigenvalue problem corresponding to different eigenvalues are $\alpha$ orthogonal. By applying the complex conjugate of the problem (21) - (23) we showed that the eigenvalues of this problem are real. Furthermore, by applying the definition of the conformable Wronskian function discovered 
the representation of the solution to the Sturm-Liouville eigenvalue problem having modified Coulomb potential. We have known that the outcomes of the solution of conformable Sturm-Liouville eigenvalue problem having modified Coulomb potential in the homogeneous case are different with distinct order of derivative, see Fig. 1 . Also, the series solutions are increasing on different positive scalar multiplication with order $\alpha=0.5$ and they are different, can see Fig. 2. The solution of a non- homogeneous case approach to negative infinite number for negative eigenvalues $\lambda$, see Fig. 4. and Fig. 6.

\section{REFERENCES}

[1] Wu, G. C., \& Baleanu. D. (2013). New applications of the variational iteration method-From differential equations to q-Fractional difference equations, Advances in Difference Equations 2013, 1-16.

[2] Bas, E., \& Metin, F. (2013). Fractional singular Sturm-Liouville operator for Coulomb potential, Advances in Difference Equations 2013, 300.

[3] Baleanu, D., \& Mustafa, O. G. (2011). On the existence interval for the initial value problem of a fractional differential equation, Hacettepe Journal of Mathematics and Statistics 40, 581-587.

[4] Cabrera, I. J., Harjani, J., \& Sadarangani, K. B. (2012). Existence and uniqueness of positive solutions for a singular fractional three-point boundary value problem, Abstract and Applied Analysis 2012.

[5] Unal, E., Gokdogan, A., \& Celik, E. (2017). Solutions around a regular $\alpha$ singular point of a sequential conformable fractional differential equation, Kuwait Journal of Science 44, 9-16.

[6] Khalil, R., \& Abu Hammad, M. (2014). Legendre fractional differential equation and Legender fractional polynomials, International Journal of Applied Mathematical Research 3, 214-219.

[7] Gökdoğan, A., Ünal, E., \& Çelik, E. (2016). Existence and uniqueness theorems for sequential linear conformable fractional differential equations, Miskolc Mathematical Notes 17, 267-279.

[8] Al-Refai, M., \& Abdeljawad, T. (2017). Fundamental results of conformable Sturm-Liouville eigenvalue problems, Complexity 2017.

[9] Bas, E., \& Acay, B. (2020). The direct spectral problem via local derivative including truncated MittagLeffler function, Applied Mathematics and Computation 367.

[10] Bas, E., Ozarslan, R.,\& Baleanu, D. (2018). Sturm-Liouville difference equations having Bessel and hydrogen atom potential type,Open Physics16, 801-809.

[11] Gökdoğan, A., Ünal, E., \& Çelik, E. (2015). Conformable fractional Bessel equation and Bessel functions, arXiv preprint arXiv:1506.07382.

[12] Baleanu, D., Mustafa, O. G., \& Agarwal, R. P. (2011). Asymptotic integration of $(1+\alpha)$-order fractional differential equations, Computers and Mathematics with Applications 62, 1492-1500.

[13] Panakhov, E. S., \& Sat, M. (2013). Reconstruction of potential function for sturm-liouville operator with coulomb potential, Boundary Value Problems 2013, 1-9.

[14] Grace, S. R., Agarwal, R. P., Wong, P. J. Y., \& Zafer, A. (2012). On the oscillation of fractional differential equations, Fractional Calculus and Applied Analysis 15, 222-231.

[15] Abdeljawad, T. (Elsevier, 2015). On conformable fractional calculus, in Journal of computational and Applied Mathematics vol. 279 57-66.

[16] Khalil, R., Al Horani, M., Yousef, A., \& Sababheh, M. (2014). A new definition of fractional derivative, Journal of Computational and Applied Mathematics 264, 65-70. 
[17] Wang, Y., Zhou, J., \& Li, Y. (2016). Fractional Sobolev's spaces on time scales via conformable fractional calculus and their application to a fractional differential equation on time scales, Advances in Mathematical Physics 2016.

[18] Bas, E., \& Najemadeen, I. (2019) "Singular eigenvalue problem with modified Frobenius method" Cmec2019 Conf. Proceed, No. 84, p. 717-726. 